\title{
The putative oncogene, CRNDE, is a negative prognostic factor in ovarian cancer patients
}

\author{
Lukasz Michal Szafron ${ }^{1, *}$, Anna Balcerak ${ }^{2,}$, Ewa Anna Grzybowska², Barbara \\ Pienkowska-Grela ${ }^{1}$, Agnieszka Podgorska ${ }^{1}$, Renata Zub ${ }^{2}$, Magdalena Olbryt ${ }^{3}$, \\ Jolanta Pamula-Pilat ${ }^{3}$, Katarzyna M. Lisowska ${ }^{3}$, Ewa Grzybowska ${ }^{3}$, Tymon Rubel ${ }^{4}$, \\ Agnieszka Dansonka-Mieszkowska ${ }^{1}$, Bozena Konopka ${ }^{1}$, Magdalena Kulesza ${ }^{1}$, \\ Martyna Lukasik ${ }^{1}$, Jolanta Kupryjanczyk ${ }^{1, *}$ \\ ${ }^{1}$ Department of Pathology and Laboratory Diagnostics, Maria Sklodowska-Curie Memorial Cancer Center and Institute of \\ Oncology, Warsaw, Poland \\ ${ }^{2}$ Department of Molecular and Translational Oncology, Maria Sklodowska-Curie Memorial Cancer Center and Institute of \\ Oncology, Warsaw, Poland \\ ${ }^{3}$ Center for Translational Research and Molecular Biology of Cancer, Maria Sklodowska-Curie Memorial Cancer Center and \\ Institute of Oncology , Gliwice Branch, Poland \\ ${ }^{4}$ Institute of Radioelectronics and Multimedia Technology, Warsaw University of Technology, Warsaw, Poland \\ * These authors have contributed equally to this work
}

Correspondence to: Lukasz Michal Szafron, e-mail: lukszafron@gmail.com

Keywords: ovarian cancer, prognostic factor, CRNDE, gene expression, TP53

Received: June 18, 2015

Accepted: October 22, 2015

Published: November 04, 2015

\section{ABSTRACT}

The CRNDE gene seems to play an oncogenic role in cancers, though its exact function remains unknown. Here, we tried to assess its usefulness as a molecular prognostic marker in ovarian cancer. Based on results of our microarray studies, CRNDE transcripts were further analyzed by Real-Time qPCR-based profiling of their expression. The qPCR study was conducted with the use of personally designed TaqMan assays on 135 frozen tissue sections of ovarian carcinomas from patients treated with platinum compounds and either cyclophosphamide $(P C, N=32)$ or taxanes (TP, $N=103$ ). Elevated levels of two different CRNDE transcripts were a negative prognostic factor; they increased the risk of death and recurrence in the group of patients treated with TP, but not PC (DNA-damaging agents only). Higher associations were found for overexpression of the short CRNDE splice variant (FJ466686): HR 6.072, 95\% CI 1.814-20.32, $p=0.003$ (the risk of death); HR 15.53, 95\% CI 3.812-63.28, $p<0.001$ (the risk of recurrence). Additionally, accumulation of the TP53 protein correlated with decreased expression of both CRNDE transcripts in tumor cells. Our results depict CRNDE as a potential marker of poor prognosis in women with ovarian carcinomas, and suggest that its significance depends on the therapeutic regimen used.

\section{INTRODUCTION}

The CRNDE (Colorectal Neoplasia Differentially Expressed, formerly known as LOC388279 or LOC643911) gene is located to the long arm of chromosome 16 (16q12.2) in human. Until recently, $C R N D E$ had been treated as a long non-coding RNA (lncRNA)-coding gene [1], though we have lately identified its protein product, CRNDEP [2]. Our interest in this gene started from its identification as one of several (and the most strongest) potential prognostic factors in ovarian cancer patients [3]. Members of our group have established complete sequences of two new CRNDE transcripts (FJ466685 and FJ466686, published in GenBank in 2008) by using the FirstChoice RLM-RACE kit (Ambion, Carlsbad, CA, USA) (see supplementary data and Figures S1-S4 in the Supplement). One of the first published studies on $C R N D E$ was an in silico experiment that used the boolean-based systems biology approach to predict novel genes associated with colorectal cancer 
[4]. The researchers identified $C R N D E$ as the gene highly upregulated $(\mathrm{FC}=16)$ in colon cancer compared with the normal colonic mucosa. Additionally, some in vitro studies showed that $C R N D E$ is overexpressed in colorectal carcinomas and other solid tumors and leukemias [5]. The authors also presented preliminary results showing that the level of CRNDE lncRNAs in patients' blood plasma increased specifically at early stages of colon cancer development. Accordingly, it was recently discovered that $C R N D E$ may promote growth and invasion of glioma cells both in vitro and in vivo [6, 7]. We have shown that the CRNDEP peptide localizes predominantly to the nucleus and its expression is elevated in highly proliferating tissues [2]. All these findings make the $C R N D E$ gene a promising candidate for a new biomarker of carcinogenesis.

Here, we aimed to elucidate how the expression of the two CRNDE transcripts identified by our team affects ovarian cancer prognosis in patients treated with two different chemotherapy regimens. Another aspect of this study was to analyze CRNDE expression with respect to TP53 accumulation status in the nuclei of tumor cells. TP53 accumulation is one of the most frequently observed aberrations in ovarian carcinomas; it occurs as a result of TP53 gene alterations that affect TP53 transactivation capabilities (mainly missense mutations) [8]. This phenomenon is predominantly due to the lack of TP53 degradation in proteasomes. Mutant TP53 exerts a dominant-negative effect on the wild-type TP53, leading to a complete loss of the TP53 function [9]. Several studies on cell lines have shown that the levels of different proteins depend on the function or level of the TP53 protein. In addition, the results obtained in recent years by our group suggest that the TP53 accumulation status may influence the clinical importance of other molecular factors [10-12]. Remarkably, this is the first study investigating a clinical importance of the $C R N D E$ gene in cancer patients.

\section{RESULTS}

\section{Evaluation of the clinical importance of the $C R N D E$ gene by gene expression microarrays, and further confirmation with real-time qPCR}

The CRNDE gene was chosen for evaluation of its prognostic value based on the results of our analysis of gene expression microarray data that are publicly available in the Gene Expression Omnibus (GEO) database (accession number GSE63885). This microarray study revealed a strong negative impact of $C R N D E$ expression on overall survival of ovarian cancer patients treated with the taxane/platinum (TP) regimen (see Table S1 in the Supplement). With FC values exceeding 5, CRNDE was the most prominent prognostic marker in that group and in its subgroup with TP53 accumulation. Moreover, this association was observed for both CRNDE-specific probe sets available in this microarray, 238021_s_at and 238022_at. A subsequent multivariate Cox analysis performed in the microarray group $(N=37)$, as well as in an adequate independent validation group $(N=66)$, confirmed the microarray outcome (see Table S2). The same statistical inference was conducted in the merged TP-treated group of 103 tumors, and the results turned out to be more significant than in either of the subgroups (see Table 1). This analysis also showed a negative impact of $C R N D E$ overexpression on disease-free survival of patients. Remarkably, the outcome obtained for the joined platinum/cyclophosphamide (PC) and TP-treated groups was, in general, less significant than for the TP-treated group only. This accorded with the lack of significant results in the univariate Cox analysis performed for the PC-treated group (data not shown).

The clinical importance of overexpressing the short CRNDE splice variant (FJ466686) was particularly visible with regard to the risk of death (HR 6.072, 95\% CI 1.814-20.32, $p=0.003$ ) and recurrence (HR 15.53, $95 \%$ CI 3.812-63.28, $p<0.001)$ in all TP-treated patients analyzed (see Table 1).

The results obtained for the longer splice variant of CRNDE (FJ466685) remained in accordance with those obtained for the shorter one, showing the negative impact of $C R N D E$ overexpression on overall survival (HR 6.908, CI 1.749-27.28, $p=0.006$ ) and disease-free survival (HR 8.760, CI 2.034-37.73, $p=0.004$ ) in the TP-treated group. However, the associations with clinical endpoints were less noticeable for the longer transcript than for the shorter one. Exemplary results are depicted in Figure 1.

Some clinical associations of $C R N D E$ appeared to be dependent on the TP53 accumulation status, which was particularly visible in the validation group (see Table S2), nevertheless, the difference could also be due to unequal sizes of subgroups. We discovered a statistically significant correlation between accumulation of the TP53 protein and the decreased expression of both $C R N D E$ transcripts in ovarian carcinomas (Mann-Whitney U test $p=0.0369$, and $p=0.0069$, for the short and long transcript, respectively, see Figure 2C-2D).

We did not find any associations between $C R N D E$ expression and the following clinicopathological parameters: patient's age, histological grade and type of a tumor, and the FIGO stage.

Lastly, we have also compared the results of CRNDE expression profiling obtained with gene expression microarrays and Real-Time qPCR, and calculated the Pearson correlation coefficients between them. The high correlation coefficients (ranging from 0.784 to 0.803 ) in combination with low $p$-values $<0.0001$ suggest that both aforementioned methods produced highly similar outcomes, which indirectly corroborates reliability of the results presented above (see Figure 2A-2B). 
Table 1: Evaluation of a prognostic value of the $C R N D E$ gene expression in the TP-treated group and the joined PC- and TP-treated groups of ovarian cancer patients.

\begin{tabular}{|c|c|c|c|c|c|c|}
\hline \multicolumn{7}{|c|}{ TP regimen } \\
\hline & & & \multicolumn{2}{|c|}{ The TP53 (+) subgroup } & \multicolumn{2}{|c|}{ The TP53 (-) subgroup } \\
\hline \multirow[b]{2}{*}{ Variable name } & OS $(87 / 103)^{*}$ & DFS $(64 / 75)^{*}$ & OS $(52 / 65)^{*}$ & DFS (41/49)* & OS $(35 / 38)^{*}$ & DFS $(23 / 26)^{*}$ \\
\hline & HR $[95 \% \mathrm{CI}] \mathrm{p}$ & HR $[95 \% \mathrm{CI}] \mathrm{p}$ & HR $[95 \% \mathrm{CI}] \mathrm{p}$ & HR $[95 \% \mathrm{CI}] \mathrm{p}$ & HR $[95 \% \mathrm{CI}] \mathrm{p}$ & HR $[95 \% \mathrm{CI}] \mathrm{p}$ \\
\hline $\begin{array}{l}C R N D E \text { (short } \\
\text { variant) high vs } \\
\text { low expr. }\end{array}$ & $\frac{6.072[1.814}{\underline{20.32] 0.003}}$ & $\frac{15.53[3.812-}{\underline{63.28]<0.001}}$ & $\frac{10.20[2.007-}{\underline{51.80] 0.005}}$ & $\frac{9.891[1.426-}{\underline{68.60] 0.020}}$ & $\begin{array}{c}5.635[0.839- \\
37.86] 0.075\end{array}$ & $\frac{18.65[1.661-}{\underline{209.3] 0.018}}$ \\
\hline $\begin{array}{l}\text { Age } \geq \mathbf{5 3} \text { vs }<53 \\
\text { years }\end{array}$ & - & $\begin{array}{c}1.648[0.938- \\
2.895] 0.082\end{array}$ & - & $\begin{array}{c}2.080[0.955- \\
4.530] 0.065\end{array}$ & - & - \\
\hline $\begin{array}{l}\text { Type (serous vs } \\
\text { non-serous) }\end{array}$ & - & $\begin{array}{c}0.446[0.216- \\
0.924] 0.030\end{array}$ & - & $\begin{array}{c}0.393[0.168- \\
0.920] 0.031\end{array}$ & $\begin{array}{c}2.371[0.869- \\
6.467] 0.092\end{array}$ & - \\
\hline Rt $>2 \mathrm{~cm}$ vs $0 \mathrm{~cm}$ & $\begin{array}{c}3.172[1.579- \\
6.371] 0.001\end{array}$ & - & $\begin{array}{c}3.052[1.298- \\
7.176] 0.011\end{array}$ & - & - & $\begin{array}{c}9.874[1.774- \\
54.97] 0.009\end{array}$ \\
\hline Rt $\leq 2 \mathrm{~cm}$ vs $0 \mathrm{~cm}$ & $\begin{array}{c}2.494[1.374- \\
4.525] 0.003\end{array}$ & $\begin{array}{c}1.875[1.079- \\
3.258] 0.026\end{array}$ & $\begin{array}{c}2.774[1.280- \\
6.010] 0.010\end{array}$ & - & - & $\begin{array}{c}2.816[0.888- \\
8.924] 0.079\end{array}$ \\
\hline $\begin{array}{l}\text { FIGO IIIA-IIIB } \\
\text { vs (IIB+IIC) }\end{array}$ & - & - & - & $\begin{array}{c}8.800[0.834- \\
92.88] 0.070\end{array}$ & - & - \\
\hline $\begin{array}{l}\text { FIGO IIIC vs } \\
(\text { IIB+IIC) }\end{array}$ & - & - & - & $\begin{array}{c}5.686[0.725- \\
44.61] 0.098\end{array}$ & - & - \\
\hline $\begin{array}{l}\text { FIGO IV vs } \\
(\text { IIB+IIC) }\end{array}$ & - & $\begin{array}{c}5.474[1.614- \\
18.56] 0.006\end{array}$ & - & $\begin{array}{c}100.4[6.951- \\
1450] 0.001\end{array}$ & - & - \\
\hline Grade 3 vs $(1+2)$ & - & $\begin{array}{c}2.168[1.177- \\
3.994] 0.013\end{array}$ & - & $\begin{array}{c}2.711[1.288- \\
5.706] 0.009\end{array}$ & - & - \\
\hline Grade 4 vs $(1+2)$ & - & - & - & - & $\begin{array}{l}9.300[2.723- \\
31.76]<0.001\end{array}$ & - \\
\hline $\begin{array}{l}C R N D E \text { (long } \\
\text { variant) high vs } \\
\text { low expr. }\end{array}$ & $\frac{6.908[1.749-}{\underline{27.28] 0.006}}$ & $\frac{8.760[2.034}{\underline{37.73] 0.004}}$ & $\frac{7.519[1.027-}{\underline{55.03] 0.047}}$ & NS & $\begin{array}{c}6.630[0.899- \\
48.93] 0.064\end{array}$ & NS \\
\hline$R t>2 \mathrm{~cm}$ vs $0 \mathrm{~cm}$ & $\begin{array}{c}3.170[1.578- \\
6.367] 0.001\end{array}$ & - & $\begin{array}{c}3.110[1.326- \\
7.293] 0.009\end{array}$ & & - & \\
\hline $\mathrm{Rt} \leq 2 \mathrm{~cm}$ vs $0 \mathrm{~cm}$ & $\begin{array}{c}2.642[1.465- \\
4.765] 0.001\end{array}$ & $\begin{array}{c}1.822[1.061- \\
3.129] 0.030\end{array}$ & $\begin{array}{c}2.984[1.381- \\
6.445] 0.005\end{array}$ & & - & \\
\hline $\begin{array}{l}\text { FIGO IV vs } \\
(\text { IIB+IIC) }\end{array}$ & - & $\begin{array}{c}4.477[1.438- \\
13.94] 0.010\end{array}$ & - & & - & \\
\hline Grade 3 vs $(1+2)$ & - & $\begin{array}{c}1.648[0.976- \\
2.782] 0.062\end{array}$ & - & & - & \\
\hline Grade 4 vs $(1+2)$ & - & - & - & & $\begin{array}{l}4.911[1.824- \\
13.227] 0.002\end{array}$ & \\
\hline
\end{tabular}

(Continued) 


\begin{tabular}{|c|c|c|c|c|c|c|}
\hline \multicolumn{7}{|c|}{$\mathrm{PC}+\mathrm{TP}$ regimens } \\
\hline & & & The TP53 ( & +) subgroup & The TP53 & -) subgroup \\
\hline \multirow[b]{2}{*}{ Variable name } & OS $(118 / 135)^{*}$ & DFS (84/97)* & OS $(72 / 86)^{*}$ & DFS $(54 / 63)^{*}$ & OS $(46 / 49)^{*}$ & DFS (30/34)* \\
\hline & HR $[95 \%$ CI $] p$ & HR $[95 \% \mathrm{CI}] \mathrm{p}$ & HR $[95 \% \mathrm{CI}] \mathrm{p}$ & HR $[95 \%$ CI] p & HR $[95 \% \mathrm{CI}] \mathrm{p}$ & HR $[95 \% \mathrm{CI}] \mathrm{p}$ \\
\hline $\begin{array}{l}C R N D E \text { (short } \\
\text { variant) high vs } \\
\text { low expr. }\end{array}$ & $\begin{array}{l}\frac{5.437[1.699-}{17.40] 0.004} \\
\underline{1}\end{array}$ & $\frac{11.47[2.869-}{\underline{45.84] 0.001}}$ & $\frac{9.454[2.099-}{\underline{42.58] 0.003}}$ & NS & NS & $\frac{22.44[2.160-}{\underline{233.3] 0.009}}$ \\
\hline $\begin{array}{l}\text { Age } \geq 53 \text { vs }<53 \\
\text { years }\end{array}$ & - & - & - & & & $\begin{array}{c}2.668[1.140- \\
6.240] 0.024\end{array}$ \\
\hline $\mathrm{Rt}>2 \mathrm{~cm}$ vs $0 \mathrm{~cm}$ & $\begin{array}{l}2.908[1.674- \\
5.051]<0.001\end{array}$ & - & $\begin{array}{l}3.325[1.696- \\
6.517]<0.001\end{array}$ & & & - \\
\hline $\mathrm{Rt} \leq 2 \mathrm{~cm}$ vs $0 \mathrm{~cm}$ & $\begin{array}{l}2.503[1.502- \\
4.169]<0.001\end{array}$ & $\begin{array}{c}1.495[0.947- \\
2.360] 0.084\end{array}$ & $\begin{array}{c}2.432[1.279- \\
4.623] 0.007\end{array}$ & & & - \\
\hline $\begin{array}{l}\text { FIGO IV vs } \\
\text { (IIB+IIC) }\end{array}$ & - & $\begin{array}{c}4.069[1.576- \\
10.51] 0.004\end{array}$ & - & & & $\begin{array}{c}9.950[1.783- \\
55.53] 0.009\end{array}$ \\
\hline Grade 3 vs $(1+2)$ & $\begin{array}{c}1.688[0.911- \\
3.129] 0.096\end{array}$ & - & - & & & - \\
\hline Grade 4 vs $(1+2)$ & $\begin{array}{c}1.853[0.947- \\
3.624] 0.072\end{array}$ & - & - & & & $\begin{array}{l}4.224[1.201- \\
14.862] 0.025\end{array}$ \\
\hline $\begin{array}{l}C R N D E \text { (long } \\
\text { variant) high vs } \\
\text { low expr. }\end{array}$ & $\begin{array}{c}2.414[0.971- \\
6.005] 0.058\end{array}$ & $\begin{array}{c}2.755[0.863- \\
8.800] 0.087\end{array}$ & $\underline{\underline{6.306[1.485-}} \underline{\underline{26.77] 0.013}}$ & NS & NS & NS \\
\hline $\begin{array}{l}\text { Age } \geq \mathbf{5 3} \text { vs }<\mathbf{5 3} \\
\text { years }\end{array}$ & - & $\begin{array}{c}1.482[0.937- \\
2.344] 0.093\end{array}$ & - & & & \\
\hline Rt $>2 \mathrm{~cm}$ vs $0 \mathrm{~cm}$ & $\begin{array}{l}2.900[1.675- \\
5.021]<0.001\end{array}$ & - & $\begin{array}{l}3.568[1.819- \\
6.998]<0.001\end{array}$ & & & \\
\hline $\mathrm{Rt} \leq 2 \mathrm{~cm}$ vs $0 \mathrm{~cm}$ & $\begin{array}{c}2.413[1.470- \\
3.958]<0.001\end{array}$ & $\begin{array}{c}1.565[0.992- \\
2.469] 0.054\end{array}$ & $\begin{array}{c}2.631[1.388- \\
4.986] 0.003\end{array}$ & & & \\
\hline $\begin{array}{l}\text { FIGO IV vs } \\
\text { (IIB+IIC) }\end{array}$ & $\begin{array}{c}1.950[1.023- \\
3.717] 0.042\end{array}$ & $\begin{array}{c}5.946[2.259- \\
15.65]<0.001\end{array}$ & - & & & \\
\hline
\end{tabular}

"Values before and after a slash (/) stand for the number of completed observations vs all observations, respectively. Only the expression results with $p$-values $<0.1$ are shown and those with $p$-values $<0.05$ are underlined. HR and CI stand for the hazard ratio and $95 \%$ confidence interval, respectively. NS - a non-significant result. The multivariate Cox proportional hazards model was utilized in this analysis.

\section{Identification of other possible splice variants of CRNDE}

Four reference CRNDE transcripts, NR_034105, NR_034106, NR_110453, and NR_110454 are spliced differently than the transcripts identified in our study (see Figure 3A). This may suggest that CRNDE pre-RNA is processed in many ways, depending on, e.g., a tissue type. As a result, a variety of distinct CRNDE RNAs could exist in different tissues. To verify this hypothesis, we carried out an additional PCR experiment on three cDNA samples derived from normal endometrium (NE), ovarian cancer (OC) and HeLa cells (He). In this study, 7 sets of primers were used (see Figure $3 \mathrm{~B}-3 \mathrm{C}$ and Table S3). The CRNDEv1F primer was specific to the NR_034105 and NR_110453 sequences, whereas CRNDEv2F should hybridize to the NR_034106 and NR_110454 transcripts only. The expected length of PCR products, depending on the primer set used and the CRNDE splice variant, is shown in Figure 3C. Although the overall band pattern was in step with our earlier predictions in silico, some unexpected bands (marked with ellipses) emerged 
in a tissue-dependent manner. On the contrary, some other bands, specific to the 5' end of the NR_034105 and NR_110453 transcripts did not appear in the gel, suggesting the lack of these splice variants in the analyzed tissues. Remarkably, the FJ466686 (shorter) transcript described herein, seemed to be present in all three samples evaluated (see double bands in Figure 3B). Accordingly, we have recently proven the prevalence of this CRNDEPcoding splice variant by measuring and comparing its expression between 21 normal human tissue sets [2].

\section{DISCUSSION}

Herein, by performing microarray and Real-Time qPCR studies, we have shown that the elevated level of $C R N D E$ transcripts significantly increases the risk of death and recurrence in ovarian cancer patients. This association was observed predominantly in patients treated with taxanes (and not in the group treated with DNAdamaging agents only), which may be a hint that this gene is somehow involved in the metabolism of microtubules during cell division. In qPCR experiments, we investigated the expression of two different splice variants of the $C R N D E$ gene. The longer variant (FJ466685) contains 83 additional nucleotides in exon 5 . Their absence in the shorter variant (FJ466686) leads to a formation of a new ORF, which encodes the 84 amino acid-peptide CRNDEP, recently described by our group as overexpressed in highly proliferating tissues [2]. This observation is concordant with the hypothesis by Ellis et al. [13], that $C R N D E$ may be a target of the MYC regulatory pathway, being associated with the cell cycle progression and malignant transformation [14]. Interestingly, our clinical results obtained for the shorter splice variant (FJ466686, CRNDEP-coding) were statistically more significant than those for the longer one, which may imply that the former variant exhibits a higher biological activity.

The clinical importance of CRNDE expression was best visible in all TP-treated patients than in either the microarray or validation subgroup. This was likely due to the relatively small size of both subgroups. Nevertheless, we obtained consistent and statistically
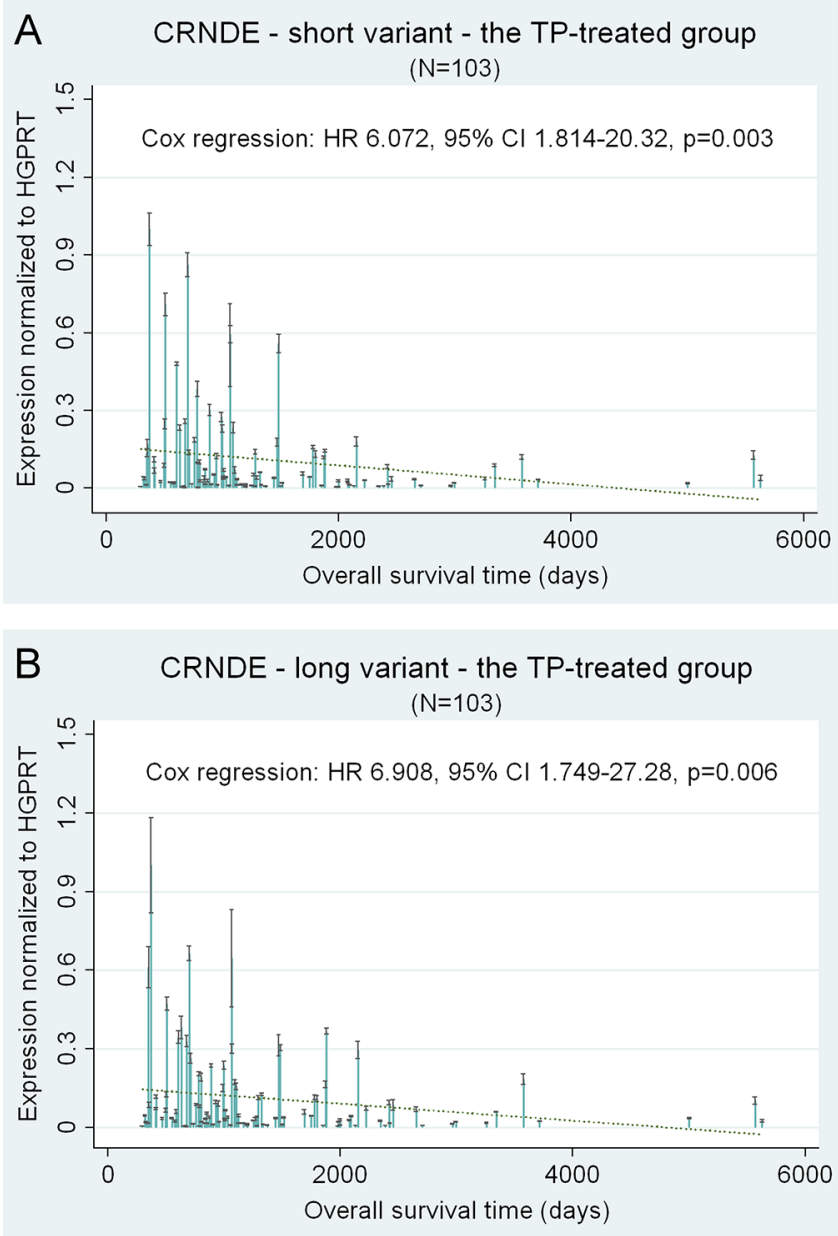

Figure 1: $C R N D E$ - selected prognostic results of the multivariate statistical analysis of gene expression. $C R N D E$ expression is shown as a continuous variable. Black lines on bars represent standard deviations of Real-Time qPCR measurements for each tumor. The linear regression lines (green and dotted) are shown to visualize trends of expression. 
significant results for all three sample sets (see Tables 1 and S2), and the overall clinical effect seemed to be additive. These results were obtained in the multivariate Cox analysis, which strongly supports the hypothesis that $C R N D E$ overexpression may be an independent, negative prognostic factor in ovarian carcinogenesis. Furthermore, the clinical significance of this novel marker seems to hinge on the therapeutic regimen used.

In accordance with our results, other researchers reported CRNDE overexpression in more than $90 \%$ of colorectal adenocarcinomas [5]. The authors also suggested that $C R N D E$ may be a promising tissue and plasma biomarker in this cancer. Noteworthy, both splice variants identified by our team were shown by Graham et al. [5] to be highly elevated in adenomas and adenocarcinomas, but not in the normal colonic tissue. The same team reported in another article [13] that $C R N D E$ may be involved in development of cancers in the variety of tissues and organs, like: colon, liver, kidney, pancreas, prostate, ovary, blood and brain. Based on some preliminary knock-in and knock-down experiments, the authors concluded that $C R N D E$ transcripts can promote carcinogenesis by stimulating cell growth and suppressing apoptosis. The rise of CRNDE levels during cancer development may be due to the alterations in upstream signaling pathways, including the MAP kinase pathway. Recently, the same authors reported the existence of a few nuclear, intronic $C R N D E$ transcripts, and found the association between expression of one of such transcripts (called gVC-In4) and the insulin/insulin-like growth factor (IGF) signaling. This mechanism seemed to depend on the activation of both the PI3K and MAPK pathways [15]. Accordingly, it was recently discovered that overexpression of the CRNDE gene may suppress apoptosis and promote growth and invasion of gliomas both in vitro and in vivo either in a manner depending on the mammalian Target of Rapamycin (mTOR) pathway [6] or by inhibiting the expression of the tumor-suppressing
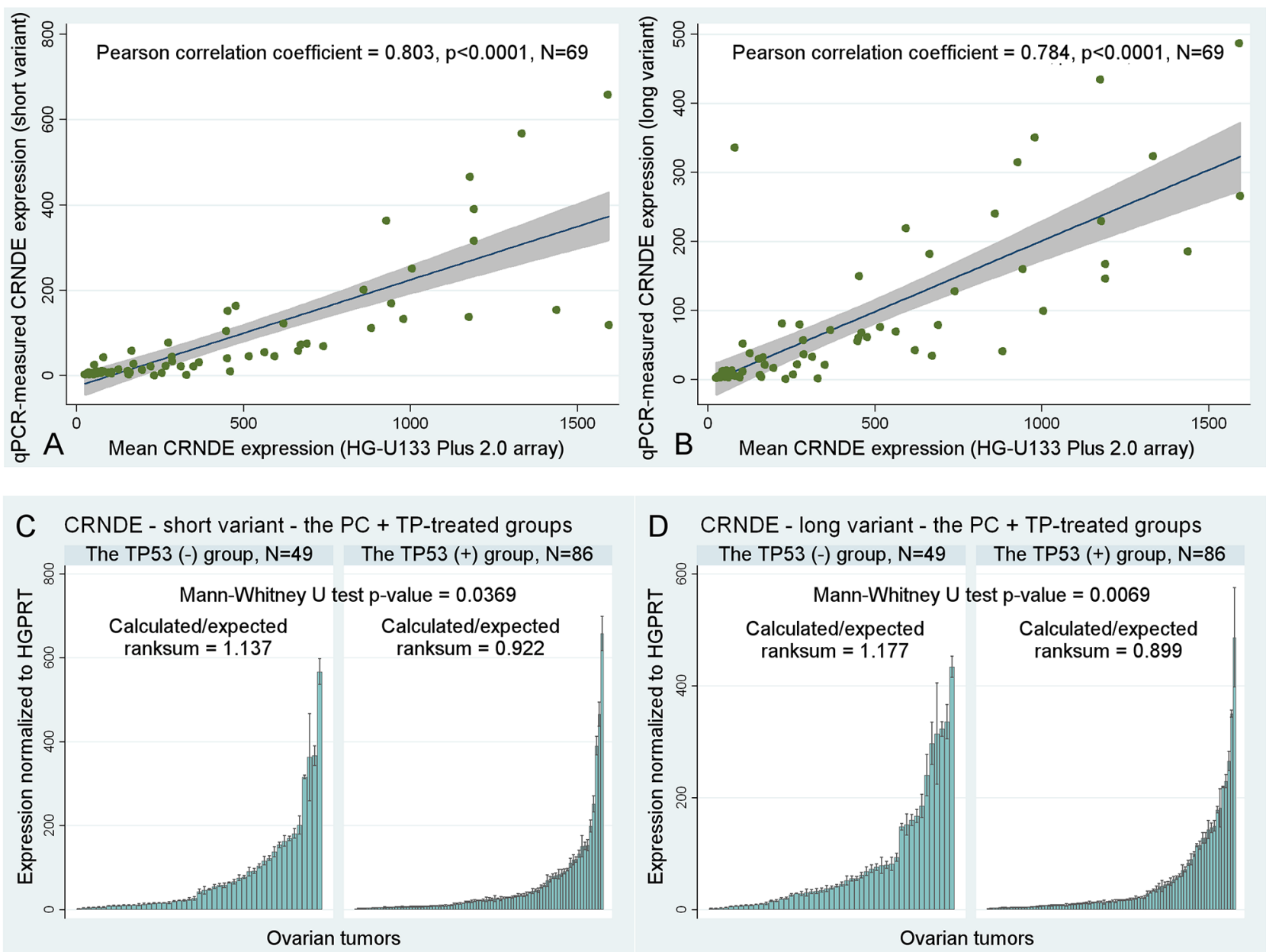

Figure 2: Other statistical results. A-B. A correlation between the mean $C R N D E$ expression measured with gene expression microarrays for two CRNDE-specific probe sets, 238021_s at and 238022_at, and the results obtained in Real-Time qPCR studies with the use of our personally designed TaqMan assays for two different splice variants of CRNDE. Fitted regression lines are marked blue, whereas shaded regions represent the $95 \%$ confidence interval. C-D. The association between the accumulation of TP53 and the decreased expression of CRNDE. If the expression decreases, the calculated/expected ratio of the Mann-Whitney U test is lower than 1 and vice versa. Black lines on bars represent standard deviations of Real-Time qPCR measurements for each tumor. 
miR-186 [7]. In line with this, Chen et al. [16] reported abundant expression of CRNDE in recurrent gliomas. All these results support our findings described herein.

We have previously shown on large groups of patients that the TP53 accumulation status determined the clinical significance of altered expression of some genes, like $B A X, B C L 2$ or $E R B B 2$, and polymorphisms in others $(A R, F S H R)$ in ovarian cancer patients $[10,11,17,18]$. This idea is based on the assumption, and some facts from studies on cell lines, that mutant TP53, in contrast to the

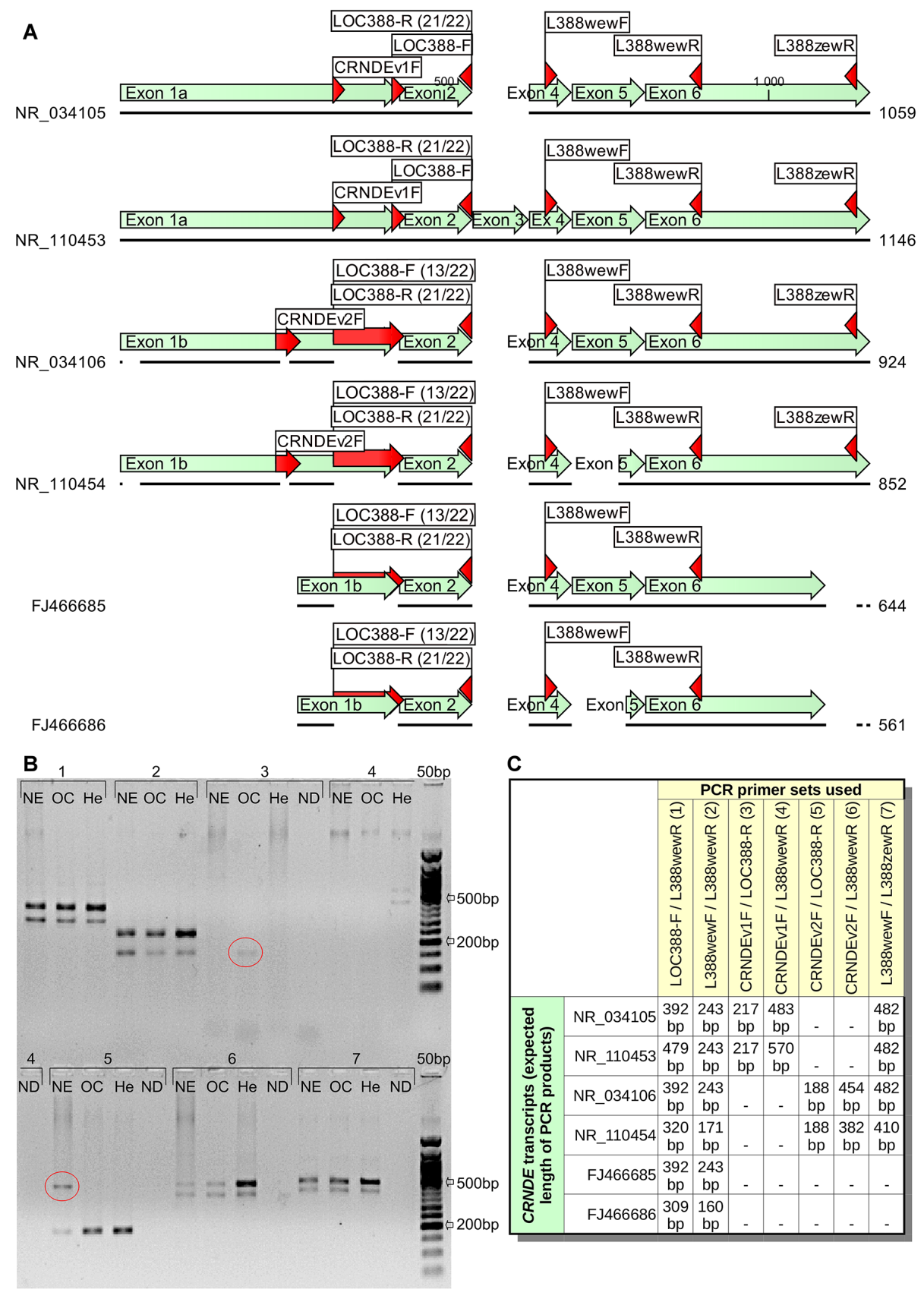

Figure 3: Different $C R N D E$ transcripts seem to occur in a tissue-dependent manner. A. A graphical alignment of two $C R N D E$ transcripts investigated in this study (FJ466685, FJ466686) to four reference RNA sequences (NR_034105, NR_034106, NR_110453, NR_110454) available in GenBank. Regions, where the PCR primers hybridize, are marked with red arrows. B. Three various tissues were tested in this PCR experiment: normal endometrium (NE), ovarian cancer (OC) and HeLa cells (He). The bands characteristic for certain tissues only and also inconsistent with in silico predictions were marked with ellipses (see Figure C for details on the primer sets used and the expected length of PCR products). 50 bp DNA Ladder (New England Biolabs, Ipswich, MA, USA) was utilized as a size standard. The annealing temperature was set to $55^{\circ} \mathrm{C}$, while the elongation time lasted for 2 minutes to allow amplification of products up to $2 \mathrm{~kb}$. ND stands for a no-DNA sample. NDs for the primer sets no. 1 and 2 were not shown due to the lack of empty lanes in the gel (they were both negative). 
wt protein, creates a "permissive" environment for action of oncogenes $[19,20]$. However, this was not apparent with regard to the $C R N D E$ gene. Concomitantly, the RealTime qPCR results presented here imply an association between TP53 accumulation and lower CRNDE expression. In accordance with this observation, Ellis et al. [13] proposed that the CRNDE and IRX5 genes may share the same bidirectional promoter, whereas the expression of IRX5 was shown to negatively correlate with mutant TP53 protein levels in LNCaP prostate cancer cells [21].

Finally, we want to present some considerations related to the methodical part of the study. In order to achieve the highest possible specificity and sensitivity of the qPCR study, we designed and tested our TaqMan assays as recommended by PE Biosystems [22]. One of the most important factors that could potentially affect the expression analysis was the presence of single-nucleotide polymorphisms (SNPs) in the regions, where the CRNDEspecific primers and the TaqMan probe bind. According to the NCBI dbSNP database, there are no SNPs in the sequences recognized by two primers, LOCrtF and LOCrt35R (see Table S3). By contrast, the LOCrt4wR primer binds to a region, where one SNP, rs575423185, has been identified in the 1000 Genomes Project [23]. The Minor Allele Frequency (MAF) of this SNP in the default global population equals $0.04 \%$. The $C R N D E$-specific TaqMan probe, that we designed, lies within a region with three known SNPs, rs572170005, rs192990372, and rs141935748. Their frequencies in the default global population equal $0.02 \%, 0.06 \%$ and $0.42 \%$, respectively. This means that the probability of being a carrier of the most frequent of these SNPs (rs141935748) in at least one allele is approximately $0.0042^{\wedge} 2+2 * 0.0042 *(1-0.0042) \approx 0.0084$. In other words, one of about $119(1 / 0.0084)$ individuals is a carrier of this SNP in the default global population. Considering that the analyzed group in our Real-Time qPCR study consisted of 135 ovarian cancer patients, it is highly probable that only one tumor harbored the rs 141935748 polymorphism, which should not affect the CRNDE expression profiling in the entire group. The 3 other SNPs mentioned above are much less frequently distributed in the default global population, so they are likely absent in our group of ovarian cancer patients.

As to the specificity of our TaqMan assays, they were designed in such a way to allow specific discrimination between the CRNDEP-coding (FJ466686) and other CRNDEP-non-coding transcripts, e.g. FJ466685. This goal was achieved by using the LOCrt35R reverse primer, specific to the CRNDEP-coding transcript only. By contrast, the LOCrtF universal forward primer as well as the TaqMan probe did not discriminate the CRNDE transcripts (see Figure 4), whereas the LOCrt4wR primer was able to detect the FJ466685 splice variant as well as all reference transcripts except one, NR_110454. Thus, our qPCR results for the CRNDEP-coding transcript are fully specific, while the results for the longer $C R N D E$ splice variant, FJ466685, are, in fact, the sum of expression for several transcripts. This is worth noting in light of the findings by Graham et al. [5], who showed that CRNDE transcripts are differentially expressed in colorectal cancer. Nevertheless, all splice variants detected by our TaqMan assays were upregulated in that neoplasm, which is consistent with the results shown herein.

As to the diversity of CRNDE transcripts in human cells, in July 2015, there were 12 different CRNDE

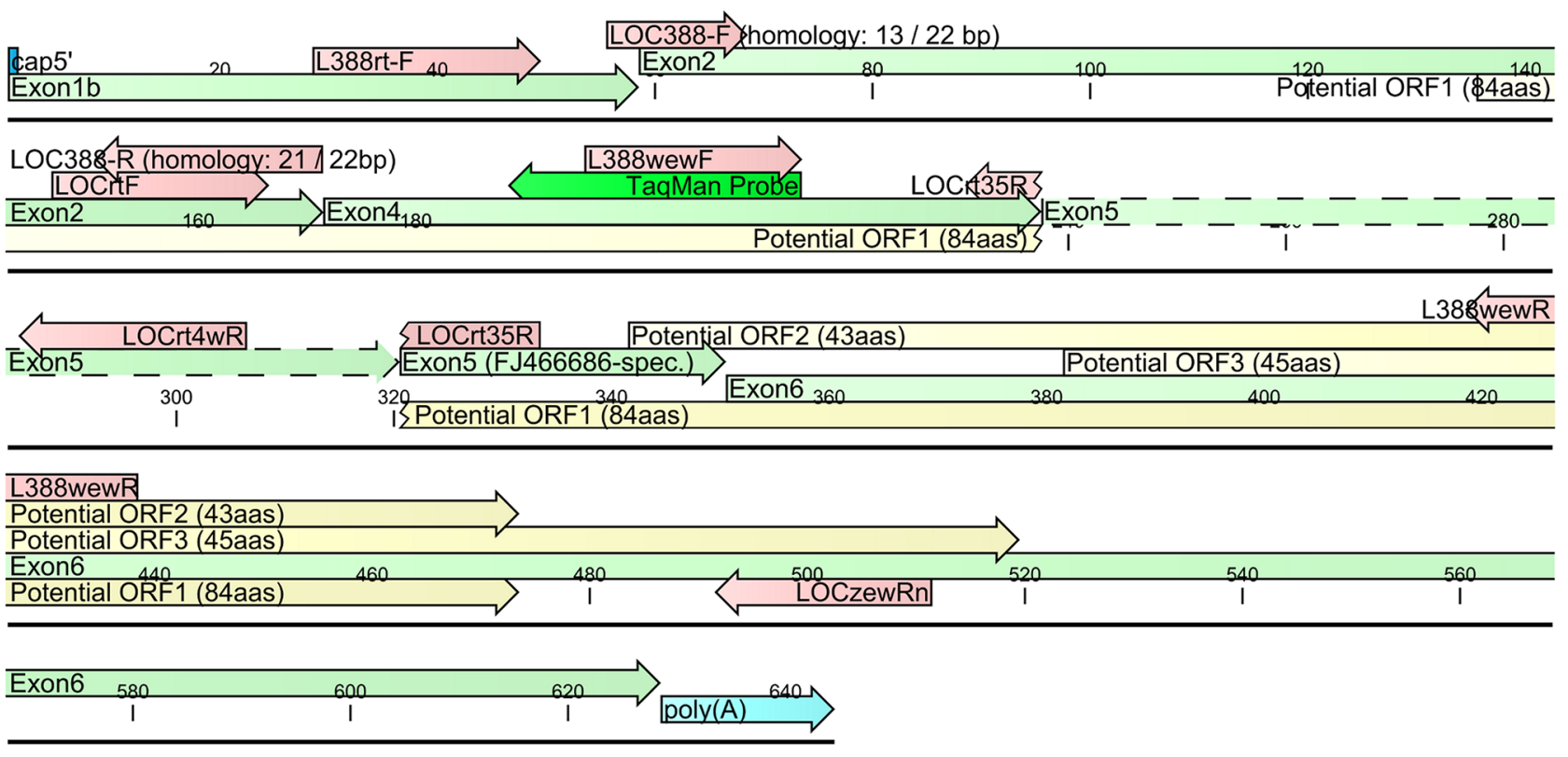

Figure 4: A diagram of two CRNDE transcripts, FJ466685 and FJ466686, investigated herein. An alternatively spliced region within exon 5 was depicted with dashed lines. The presence of this region leads to a disruption of 84aa ORF encoding the CRNDEP peptide. Red arrows represent primer sequences. 
Table 2: Patients' characteristics

\begin{tabular}{|c|c|c|c|c|}
\hline & \multicolumn{3}{|c|}{ Microarray group } & \multirow{2}{*}{$\begin{array}{c}\text { Validation group } \\
\text { TP regimen } \\
(N=66)\end{array}$} \\
\hline & $\begin{array}{l}\text { PC regimen } \\
\quad(N=32)\end{array}$ & $\begin{array}{l}\text { TP regimen } \\
\qquad(N=37)\end{array}$ & $\begin{array}{c}\text { PC+TP regimens } \\
(N=69)\end{array}$ & \\
\hline \multicolumn{5}{|l|}{$\begin{array}{l}\text { Age of patients } \\
\text { (years) }\end{array}$} \\
\hline Range (median) & $34-68(54)$ & $32-74(53)$ & $32-74(53)$ & $20-79(53)$ \\
\hline \multicolumn{5}{|l|}{ Histological type } \\
\hline Serous & $31(96.9 \%)$ & $30(81.1 \%)$ & $61(88.4 \%)$ & $49(74.2 \%)$ \\
\hline Endometrioid & - & $2(5.4 \%)$ & $2(2.9 \%)$ & $2(3.0 \%)$ \\
\hline Clear-cell & - & - & - & $3(4.6 \%)$ \\
\hline Undifferentiated & - & $4(10.8 \%)$ & $4(5.8 \%)$ & $4(6.1 \%)$ \\
\hline Other types & $1(3.1 \%)$ & $1(2.7 \%)$ & $2(2.9 \%)$ & $8(12.1 \%)$ \\
\hline \multicolumn{5}{|l|}{ Histological grade } \\
\hline $\mathrm{G} 1, \mathrm{G} 2$ & $4(12.5 \%)$ & $5(13.5 \%)$ & $9(13.0 \%)$ & $9(13.6 \%)$ \\
\hline G3 & $18(56.3 \%)$ & $23(62.2 \%)$ & $41(59.4 \%)$ & $37(56.1)$ \\
\hline G4 & $10(31.3 \%)$ & $9(24.3 \%)$ & $19(27.5 \%)$ & $20(30.3 \%)$ \\
\hline \multicolumn{5}{|c|}{ Clinical stage (FIGO) } \\
\hline IIB, IIC & - & $2(5.4 \%)$ & $2(2.9 \%)$ & $1(1.5 \%)$ \\
\hline IIIA, IIIB & $6(18.8 \%)$ & $5(13.5 \%)$ & $11(15.9 \%)$ & $4(6.1 \%)$ \\
\hline IIIC & $22(68.8 \%)$ & $25(67.6 \%)$ & $47(68.1 \%)$ & $59(89.4 \%)$ \\
\hline IV & $4(12.5 \%)$ & $5(13.5 \%)$ & $9(13.0 \%)$ & $2(3.0 \%)$ \\
\hline \multicolumn{5}{|l|}{ Residual tumor size } \\
\hline $0 \mathrm{~cm}$ & $8(25.0 \%)$ & $7(18.9 \%)$ & $15(21.7 \%)$ & $18(27.7 \%)^{*}$ \\
\hline$\leq 2 \mathrm{~cm}$ & $9(28.1 \%)$ & $23(62.2 \%)$ & $32(46.4 \%)$ & $35(53.9 \%)$ \\
\hline$>2 \mathrm{~cm}$ & $15(46.9 \%)$ & $7(18.9 \%)$ & $22(31.9 \%)$ & $12(18.5 \%)$ \\
\hline \multicolumn{5}{|l|}{$\begin{array}{l}\text { Overall survival } \\
\text { (days) }\end{array}$} \\
\hline Range (median) & $104-3750(1165.5)$ & $357-5630$ (1069) & $104-5630(1111)$ & $296-5569$ (1161.5) \\
\hline \multicolumn{5}{|c|}{$\begin{array}{l}\text { Disease free survival } \\
\text { (days) }\end{array}$} \\
\hline Range (median) & $97-2521(369.5)$ & $116-2452(448)$ & $97-2521(416)$ & 96-2884 (490) \\
\hline \multicolumn{5}{|l|}{ Outcome } \\
\hline NED & $1(3.1 \%)$ & $3(8.1 \%)$ & $4(5.8 \%)$ & $10(15.2 \%)$ \\
\hline AWD & - & - & - & $3(4.6 \%)$ \\
\hline DOD & $31(96.9 \%)$ & $34(91.9 \%)$ & $65(94.2 \%)$ & $53(80.3 \%)$ \\
\hline \multicolumn{5}{|l|}{ TP53 accumulation } \\
\hline TP53(-) & $11(34.4 \%)$ & $14(37.8 \%)$ & $25(36.2 \%)$ & $24(36.4 \%)$ \\
\hline TP53(+) & $21(65.6 \%)$ & $23(62.2 \%)$ & $44(63.8 \%)$ & $42(63.6 \%)$ \\
\hline
\end{tabular}

NED - no evidence of disease; AWD - alive with disease; DOD - died of disease.

*The information on a residual tumor size for one patient from the validation group is missing. 
mRNAs collected in the AceView database, the length of which ranged from $101 \mathrm{bp}$ (FN117285, identified in breast carcinoma) to $2221 \mathrm{bp}$ (the variant reconstructed from 25 cDNA clones originating from various normal and cancerous tissues). Here, we compared the splice variants described by us with four reference transcripts, NR_034105, NR_034106, NR_110453, and NR_110454. Although our $\bar{C} R N D E$ transcripts were capped and polyadenylated, which suggested their completeness, the alignment showed that they were shorter on both ends than the reference sequences. The boundaries of exons 1a and $1 \mathrm{~b}$ described by Graham et al. [5] represent the most external transcription initiation sites, however, the most common initiation sites are located nearer the $3^{\prime}$ ends of these exons. Accordingly, the $5^{\prime}$ end of the transcripts cloned by our group was among those mapped by $5^{\prime}$ RACE in colorectal cancer cell lines by Graham et al. [5]. As to the $3^{\prime}$ end of $C R N D E$ transcripts, the polyadenylation site identified by our group was also the one, which was the most commonly observed by Graham et al., though in the reference sequences the most external polyadenylation site was indicated [5]. Consistently, one of our bacterial clones, harboring the products of $3^{\prime} \mathrm{RACE}$ for $C R N D E$, had the $3^{\prime}$ UTR longer by 5 bases than the others, (see Figure S1). Moreover, the presence of unexpected PCR products in Figure $3 \mathrm{~B}$ suggests that some CRNDE transcripts in normal endometrium and ovarian cancer cells are likely spliced in a different, currently undescribed way. This supports the hypothesis that in these cells alternative termination of transcription may occur, just like in colorectal cancer cells investigated by Graham et al. [5]. Similarly, the 5' UTRs of our transcripts may also be longer, considering the positive PCR results with the use of the CRNDEv2F primer (see Figure 3). This fact implies that $C R N D E$ transcription in normal endometrium, ovarian cancer and HeLa cells starts at different initiation sites. Remarkably, we did not obtain expected PCR products for normal endometrium and ovarian cancer when using the CRNDEv1F primer, specific to exon 1a. The lack of amplification may suggest that transcripts containing this particular exon are not expressed in these tissues. On the other hand, the CRNDEv1F primer binds upstream of the main start sites identified by Graham et al. [5], so the existence of transcripts starting nearer the $3^{\prime}$ end of exon la cannot be excluded.

In summary, CRNDE seems to play a prominent role in carcinogenesis. While this study is the first demonstration of the clinical importance of $C R N D E$ in cancer patients, other researchers also reported its oncogenic role in gliomas $[6,7,16]$ and other solid tumors and leukemias [13]. Furthermore, $C R N D E$ transcripts were implied to be of potential use as sensitive and specific molecular markers of early carcinogenesis in colon cancer [5]. With all this considered, this gene is likely to become in the future an important marker of cancer development or even a target for therapy.

\section{MATERIALS AND METHODS}

\section{Patients and tumors}

The first step of our study was the cDNA microarray-based evaluation of gene expression carried out in groups of 32 and 37 frozen tumor samples from ovarian cancer patients who underwent the cisplatin/ cyclophosphamide (PC) or taxane/cisplatin (TP) treatment (see Table 2 for a detailed clinicopathological characteristics). Noteworthy, the microarray subgroup was selected from a larger tumor collection in such a way to minimize the influence of clinical factors on patients' outcome. In other words, this group comprised the patients who had a good prognosis despite a large residual tumor (being a strong, negative prognostic factor), and vice versa. Next, we used Real-Time qPCR to verify these results in the same group of tumors. We also evaluated the clinical importance of CRNDE expression on 66 previously untested ovarian tumors from patients treated with TP, which gives 135 frozen tumor samples in total. All of them were collected prior to the chemical treatment in the Institute of Oncology, Warsaw, Poland in years 1995-2010. Medical records of all patients were critically reviewed by at least two clinicians. The material was carefully selected to meet the following criteria: no chemotherapy before staging laparotomy, adequate staging procedure, International Federation of Gynecologists and Obstetricians (FIGO) stage IIB to IV disease [24], tumor tissue from the first laparotomy available, moderate or poor tumor differentiation, availability of clinical data including residual tumor size and follow-up.

All tumors were uniformly reviewed histopathologically, classified according to the criteria of the World Health Organization [25] and graded in a four-grade scale, in compliance with the standards given by Barber et al. [26]. Additionally, a complete evaluation of TP53 status has been performed using the PAb1801 mouse monoclonal antibody (1:500, SigmaGenosys, Cambridge, UK), as described previously [10]. Accumulation of TP53 in immunohistochemical staining results predominantly from missense mutations (they account for about $67 \%$ of all TP53 alterations). In case of other mutations in the gene and when no alterations occur, accumulation of the TP53 protein is not observed [27]. From among 135 specimens that we examined, the TP53 accumulation occurred in 86 tumors (63.7\%), while 49 tumors $(36.3 \%)$ were TP53-negative.

As to the evaluation of clinical endpoints, complete remission (CR) was defined as disappearance of all clinical and biochemical symptoms of ovarian cancer evaluated after completion of the first-line chemotherapy and confirmed four weeks later [28]. Disease-free survival (DFS) time was assessed only for patients who achieved complete remission. For the PC-treated group, 
the follow-up time ranged from 104 to 3750 days (the median equaled 1165.5 days); the respective values for the TP-treated group were 296 and 5630 days (with the median of 1105 days). All surviving patients had at least a 6-month follow-up.

\section{RNA extraction followed by the assessment of its quantity and quality}

RNA was isolated from frozen tumor sections with stromal cell contamination (scc) lower than 15\% utilizing the RNeasy Plus Mini Kit (Qiagen, Hilden, Germany). RNA quantity was measured with NanoDrop specrophotometer (Thermo-Fisher Scientific, Waltham, MA, USA), and its quality was assessed on Agilent Bioanalyzer (Agilent Technologies, Santa Clara, CA, USA). RNA integrity numbers (RINs) of the samples ranged from 6.5 to 9.4 .

\section{A microarray analysis of gene expression}

Hybridizations were carried out as described by Lisowska et al., [29]. Briefly, double-stranded cDNA was synthesized with Reverse Transcriptase II (Life Technologies, Carlsbad, CA, USA) using $8 \mu \mathrm{g}$ of total RNA as a template. Obtained cDNA (16 $\mu \mathrm{g})$ was then used for the synthesis of biotinylated cRNA with the BioArray High Yield RNA Transcript Labeling Kit (Enzo Life Sciences, Farmingdale, NY, USA). Both cDNA and cRNA were purified with GeneChip Sample Cleanup Module (Affymetrix, Santa Clara, CA, USA). Fragmented cRNA was hybridized first to the Affymetrix control Test3 Array, and then, after evaluating the samples' quality, to the Human Genome U133 Plus 2.0 Array (Affymetrix) for $16 \mathrm{~h}$ at $45^{\circ} \mathrm{C}$. The microarrays were stained, washed, and subsequently scanned with GeneChip Scanner 3000 (Affymetrix). Data were acquired using the GCOS 1.2 software (Affymetrix).

\section{PCR experiments}

All polymerase chain reactions (PCRs) were run on cDNA using the AmpliTaq Gold DNA Polymerase (Life Technologies). They consisted of the following steps: $95^{\circ} \mathrm{C}$ for $10 \mathrm{~min}$. (hot start of the polymerase), $95^{\circ} \mathrm{C}$ for $30 \mathrm{sec}$. (DNA denaturation), $55^{\circ} \mathrm{C}$ for $30 \mathrm{sec}$. (primers annealing), $72^{\circ} \mathrm{C}$ for $1 \mathrm{~min}$. (the PCR product elongation), $72^{\circ} \mathrm{C}$ for $7 \mathrm{~min}$. (final elongation of the product), $4^{\circ} \mathrm{C}$ hold. DNA denaturation, primers annealing and product elongation steps were repeated consecutively 35 times. The ND (no-DNA) sample was used as a negative reference in every PCR reaction. Sequences of all PCR primers are shown in Table S3.

\section{Development of two TaqMan assays for two different splice variants of $C R N D E$}

Two different sets of primers (LOCrtF, LOCrt35R) and (LOCrtF, LOCrt4wR) as well as one TaqMan probe were designed and tested, according to PE Biosystems' recommendations. These assays were able to specifically detect either the short or the long splice variant of CRNDE (see Figure S5), though the same TaqMan probe (27 bp) was utilized in both cases. This was achieved by using either the LOCrt35R or LOCrt4wR reverse primer. The former primer spanned a junction between exon 4 and the shorter splice variant of exon 5, which made it specific to the FJ466686 transcript. On the contrary, the LOCrt4wR primer was fully homologous to exon 5 in a region present in FJ466685 and absent in the FJ466686 transcript (see Figure 4). The genomic sequence of $C R N D E$ was undetectable by both primer sets due to the presence of large introns $(>4 \mathrm{~kb})$ between the amplified exons.

Both personally designed TaqMan assays specific to the CRNDE gene were thoroughly tested before use, as suggested by PE Biosystems [22], to ensure the best specificity and performance of Real-Time qPCR. Results of the specificity tests are presented in Figure S5, whereas the performance of each assay equaled about $100 \%$ (assessed based on a slope of the standard curve). In addition, the correctness of both Real-Time qPCR products was confirmed by DNA sequencing with the use of BigDye Terminator v3.1 Cycle Sequencing Kit (Life Technologies).

\section{Real-time qPCR-based studies of gene expression}

Gene expression data obtained from cDNA microarrays were further verified by Real-Time qPCR. This study was performed not only in the microarray group, but also on another set of 66 previously untested ovarian tumors (so-called the validation group) in order to confirm a clinical significance of the results. All Real-Time qPCR experiments described here were run in triplicates on the 7500 Fast Real-Time PCR System (Life Technologies), using HGPRT as a reference gene. Gene expression was evaluated with TaqMan assays, $C R N D E$-specific (6-FAM-labeled, personally designed) and HGPRT-specific (VIC-labeled, Life Technologies, assay id: 4326321E). All qPCR experiments were carried out as standard singleplex reactions in the volume of $10 \mu \mathrm{l}$ using TaqMan Universal PCR Master Mix with uracil N-glycosylase (Life Technologies) and about 20 ng of total RNA, earlier reverse transcribed to cDNA with the High-Capacity cDNA Reverse Transcription Kit (Life Technologies). The expression of CRNDE was normalized to $H G P R T$ and relatively quantified using the delta-deltaCT algorithm [30]. 
The reference gene used in this study, HGPRT, was nominated from among 11 genes included on TaqMan Human Endogenous Control Plates (Life Technologies), because it was characterized by the most stable expression in both the PC-and TP-treated groups. Expression of the reference genes was assessed for 8 randomly selected tumors from each group. Then, the stability was calculated with the qBase ${ }^{\text {PLUS }}$ software (Biogazelle NV, Zwijnaarde, Belgium), utilizing an improved version of the geNorm algorithm [31, 32].

\section{Statistical analyses of gene expression data}

Microarray data were subjected to a statistical analysis utilizing the GC-RMA algorithm [33]. Further noise reduction was achieved by the elimination of the least significant principal components. Quality control parameters were based on MAS5.0-extracted information, using thresholds suggested by Affymetrix [34]. Internal consistency of the data sets was also tested using the principal component analysis (PCA). A selection of differentially expressed genes was carried out using the resampling-based test with t statistics. Probe sets with $p$-values $\leq 0.001$ and fold change (FC) values $\geq 1.5$ were considered significantly changed.

A prognostic value of the data obtained in microarray and qPCR experiments was further verified using the stepwise multivariate Cox proportional hazards model. In this analysis, the following 6 variables were taken into account: gene expression level, patient's age (categorized by median split); residual tumor size; clinical stage (FIGO); histological grade (the last three parameters were categorized as shown in Table 2) and histological type (categorization: serous vs non-serous types). The survival analyses were performed not only in the entire group of tumors, but also in subgroups with and without accumulation of TP53. Since the PC-treated group was too small for a multivariate analysis, the univariate Cox proportional hazards model was applied to it. In addition, we conducted the statistical inference in the joined PC+TP-treated groups to find out whether the outcome depends on the therapeutic regimen used, and if this effect is additive or subtractive.

The association of $C R N D E$ expression with either clinicopathological parameters or the TP53 status in the tumors was assessed using the Mann-Whitney $U$ test or the Kruskal-Wallis test, respectively, depending whether the nominal variable had two or more categories.

Herein, the expression of CRNDE was always treated as a continuous variable to avoid arbitrary categorization of data, which could potentially lead to falsification of statistical results. It is worth noting that, in case of continuous variables, HRs cannot be treated as the ratio of the hazard rates, corresponding to the conditions described by two sets of explanatory variables (as for categorical variables), because these sets do not exist. For continuous variables, the same interpretation applies to a unit difference [35]. In our study, a tumor exhibiting the highest expression of each $C R N D E$ transcript was used as a calibrator. Thus, all the expression values ranged from 0 to 1 . This approach allowed for approximate estimation of the risk of death and recurrence based on the hazard ratio (HR) values in a similar way as for categorical variables. However, it has to be stressed that the real HR will always be lower from what is shown in Tables 1 and $\mathrm{S} 2$, because only one tumor (calibrator) has the CRNDE expression equaling 1 , and none - equaling 0 .

\section{CONFLICTS OF INTEREST}

The authors have no conflict of interests to disclose.

\section{GRANT SUPPORT}

This study was supported by the grants no. $\mathrm{N}$ N401 2361 34, N N407 0272 38, 3 P05A 060 25, and PBZ-KBN-091/P05/56 of the Polish Ministry of Science and Higher Education. The funders had no role in study design, data collection and analysis, decision to publish, or preparation of the manuscript.

\section{REFERENCES}

1. Cabili MN, Trapnell C, Goff L, Koziol M, Tazon-Vega B, Regev A, Rinn JL. Integrative annotation of human large intergenic noncoding RNAs reveals global properties and specific subclasses. Genes Dev. 2011; 25:1915-27.

2. Szafron LM, Balcerak A, Grzybowska EA, PienkowskaGrela B, Felisiak-Golabek A, Podgorska A, Kulesza M, Nowak N, Pomorski P, Wysocki J, Rubel T, DansonkaMieszkowska A, Konopka B, et al. The Novel Gene CRNDE Encodes a Nuclear Peptide (CRNDEP) Which Is Overexpressed in Highly Proliferating Tissues. PloS One. 2015; 10:e127475.

3. Szafron L, Lisowska K, Rubel T, Sobiczewski P, Kupryjanczyk J. ING1, PTPN2, PCID2, VGLL1 and LOC388279 genes as potential prognostic markers in ovarian cancer. Int J Mol Med. 2009; 24:S40.

4. Nagaraj SH, Reverter A. A Boolean-based systems biology approach to predict novel genes associated with cancer: Application to colorectal cancer. BMC Syst Biol. 2011; 5:35.

5. Graham LD, Pedersen SK, Brown GS, Ho T, Kassir Z, Moynihan AT, Vizgoft EK, Dunne R, Pimlott L, Young GP, Lapointe LC, Molloy PL. Colorectal Neoplasia Differentially Expressed (CRNDE), a Novel Gene with Elevated Expression in Colorectal Adenomas and Adenocarcinomas. Genes Cancer. 2011; 2:829-40.

6. Wang Y, Wang Y, Li J, Zhang Y, Yin H, Han B. CRNDE, a long-noncoding RNA, promotes glioma cell growth and invasion through mTOR signaling. Cancer 
Lett [Internet]. 2015; Available from: http://dx.doi. org/10.1016/j.canlet.2015.03.027

7. Zheng J, Li X-D, Wang P, Liu X-B, Xue Y-X, Hu Y, Li Z, Li Z-Q, Wang Z-H, Liu Y-H. CRNDE affects the malignant biological characteristics of human glioma stem cells by negatively regulating miR-186. Oncotarget. 2015; 6:2533955. doi: 10.18632/oncotarget.4509.

8. Blagosklonny MV. Loss of function and p53 protein stabilization. Publ Online 14 Oct 1997 Doi101038sjonc1201374 [Internet]. 1997 [cited 2015 Sep 18]; 15. Available from: http://www.nature.com/onc/journal/v15/n16/abs/1201374a. html

9. Blagosklonny MV. p53 from complexity to simplicity: mutant p53 stabilization, gain-of-function, and dominantnegative effect. FASEB J. 2000; 14:1901-7.

10. Kupryjanczyk J, Szymanska T, Madry R, Timorek A, Stelmachow J, Karpinska G, Rembiszewska A, Ziolkowska I, Kraszewska E, Debniak J, Emerich J, Ulanska M, Pluzanska A, et al. Evaluation of clinical significance of TP53, BCL-2, BAX and MEK1 expression in 229 ovarian carcinomas treated with platinum-based regimen. Br J Cancer. 2003; 88: 848-54.

11. Kupryjanczyk J, Madry R, Plisiecka-Halasa J, Bar J, Kraszewska E, Ziolkowska I, Timorek A, Stelmachow J, Emerich J, Jedryka M, Pluzanska A, Rzepka-Gorska I, Urbanski K, et al. TP53 status determines clinical significance of ERBB2 expression in ovarian cancer. Br J Cancer. 2004; 91: 1916-23.

12. Felisiak-Golabek A, Rembiszewska A, Rzepecka IK, Szafron L, Madry R, Murawska M, Napiorkowski T, Sobiczewski P, Osuch B, Kupryjanczyk J, the Polish Ovarian Cancer Study Group (P. O. C. S. G). Nuclear survivin expression is a positive prognostic factor in taxane-platinum-treated ovarian cancer patients. J Ovarian Res. 2011; 4:20.

13. Ellis BC, Molloy PL, Graham LD. CRNDE: A Long NonCoding RNA Involved in CanceR, Neurobiology, and DEvelopment. Front Genet. 2012; 3:270.

14. Wahlstrom T, Arsenian Henriksson M. Impact of MYC in regulation of tumor cell metabolism. Biochim Biophys Acta [Internet]. 2014; Available from: http://dx.doi. org/10.1016/j.bbagrm.2014.07.004

15. Ellis BC, Graham LD, Molloy PL. CRNDE, a long noncoding RNA responsive to insulin/IGF signaling, regulates genes involved in central metabolism. Biochim Biophys Acta. 2014; 1843:372-86.

16. Chen Y, Wu J-J, Lin X-B, Bao Y, Chen Z-H, Zhang C-R, Cai Z, Zhou J-Y, Ding M-H, Wu X-J, Sun W, Qian J, Zhang L, et al. Differential lncRNA expression profiles in recurrent gliomas compared with primary gliomas identified by microarray analysis. Int J Clin Exp Med. 2015; 8:5033-43.

17. Ziolkowska-Seta I, Madry R, Kraszewska E, Szymanska T, Timorek A, Rembiszewska A, Kupryjanczyk J. TP53,
BCL-2 and BAX analysis in 199 ovarian cancer patients treated with taxane-platinum regimens. Gynecol Oncol. 2009; 112:179-84.

18. Ludwig AH, Murawska M, Panek G, Timorek A, Kupryjanczyk J. Androgen, progesterone, and FSH receptor polymorphisms in ovarian cancer risk and outcome. Endocr Relat Cancer. 2009; 16:1005-16.

19. Kupryjanczyk J. Why TP53 status does not associate with clinical endpoints in ovarian cancer: Facts and hypotheses. Gynecol Oncol. 2006; 100:5-7.

20. Huang GC, Hobbs S, Walton M, Epstein RJ. Dominant negative knockout of p53 abolishes ErbB2-dependent apoptosis and permits growth acceleration in human breast cancer cells. Br J Cancer. 2002; 86:1104-9.

21. Myrthue A, Rademacher BLS, Pittsenbarger J, KutybaBrooks B, Gantner M, Qian DZ, Beer TM. The iroquois homeobox gene 5 is regulated by 1,25-dihydroxyvitamin D3 in human prostate cancer and regulates apoptosis and the cell cycle in LNCaP prostate cancer cells. Clin Cancer Res Off J Am Assoc Cancer Res. 2008; 14:3562-70.

22. PE Biosystems. Sequence Detection Systems Quantitative Assay Design and Optimization [Internet]. Available from: http://www.gene-quantification.de/pe-realtimeoverview-2.pdf

23. 1000 Genomes Project Consortium, Abecasis GR, Altshuler D, Auton A, Brooks LD, Durbin RM, Gibbs RA, Hurles ME, McVean GA. A map of human genome variation from population-scale sequencing. Nature. 2010; 467:1061-73.

24. Creasman WJ. Announcement, FIGO stages 1988, revisions. Gynecol Oncol. 1989; 35:125-7.

25. Tavassoli FA, Devilee P. Pathology and genetics of tumours of the breast and female genital organs World Health Organisation classification of tumours. IARC Press Lyon. 2003;

26. Barber HR, Sommers SC, Synder R, Kwon TH. Histologic and nuclear grading and stromal reactions as indices for prognosis in ovarian cancer. Am J Obstet Gynecol. 1975; 121:795-807.

27. Dansonka-Mieszkowska A, Ludwig AH, Kraszewska E, Kupryjanczyk J. Geographical variations in TP53 mutational spectrum in ovarian carcinomas. Ann Hum Genet. 2006; 70:594-604.

28. Miller AB, Hoogstraten B, Staquet M, Winkler A. Reporting results of cancer treatment. Cancer. 1981; 47:207-14.

29. Lisowska KM, Dudaladava V, Jarzab M, Huzarski T, Chmielik E, Stobiecka E, Lubinski J, Jarzab B. BRCA1related gene signature in breast cancer: the role of ER status and molecular type. Front Biosci Elite Ed. 2011; 3:125-36.

30. Livak KJ, Schmittgen TD. Analysis of relative gene expression data using real-time quantitative PCR and the 2(-Delta Delta C(T)) Method. Methods San Diego Calif. $2001 ; 25: 402-8$. 
31. Vandesompele J, De Preter K, Pattyn F, Poppe B, Van Roy N, De Paepe A, Speleman F. Accurate normalization of real-time quantitative RT-PCR data by geometric averaging of multiple internal control genes. Genome Biol. 2002; 3:RESEARCH0034.

32. Hellemans J, Mortier G, De Paepe A, Speleman F, Vandesompele J. qBase relative quantification framework and software for management and automated analysis of real-time quantitative PCR data. Genome Biol. 2007; 8:R19.
33. Wu Z, Irizarry RA, Gentleman R, Murillo FM, Spencer F. A model-based background adjustment for oligonucleotide expression arrays. J Am Stat Assoc. 2004; 99:909-917.

34. Affymetrix. GeneChip ${ }^{\circledR}$ Expression Analysis - Data Analysis Fundamentals. 2002.

35. Altman DG. Practical Statistics for Medical Research. Chapman and Hall; 1991. 